\title{
Infrared Bounds and the Peierls Argument in Two Dimensions ${ }^{\star}$
}

\author{
Jean Bricmont and Jean-Raymond Fontaine
}

Institut de Physique Théorique, Université Catholique de Louvain, Chemin du Cyclotron, B-1348 Louvain-la-Neuve, Belgium

\begin{abstract}
We propose a definition of contours for spin systems which leads to improved estimates on the region of parameters where several phases coexist. We discuss as examples anisotropic rotators and a $\lambda \phi^{4}$ lattice field theory. Our contours are estimated using infrared bounds and they are related to those of Euclidean Field Theory.
\end{abstract}

\section{Introduction}

The purpose of this paper is to present improved estimates on the region of parameter space where phase coexistence takes place. We discuss few examples but the method based on a new (at least in statistical mechanics) definition of contours may have a wider range of applicability.

Specifically we first consider (Sect. 2) an anisotropic rotator model in two dimensions :

$$
-H=\sum_{\langle x y\rangle} \mathbf{S}_{x} \cdot \mathbf{S}_{y}+\alpha \sum_{\langle x y\rangle} S_{x}^{1} S_{y}^{1} .
$$

It is known $[9,13,14]$ that for any $\alpha \neq 0$ there is a spontaneous magnetisation for $\beta$ large enough. However, as $\alpha$ goes to zero, $\beta$ has to be taken of order $\alpha^{-1}$ (at least). Heuristic arguments indicate that this is not the actual behaviour of $\alpha_{\text {crit }}(\beta)$ : as $\beta$ goes to infinity $\alpha_{\text {crit }}(\beta)$ should behave like $\exp (-c \beta)$ except for the case of two components where, due to the Kosterlitz-Thouless transition in the $\alpha=0$ case [11], $\alpha_{\text {crit }}(\beta)$ should reach zero for finite $\beta$.

We prove that for some $c>0$, if $\alpha=\exp (-c \beta)$ then for all $\beta$ large enough, the model (1) exhibits a spontaneous magnetisation. However our constant $c$ is not best possible.

\footnotetext{
* Part of this work was done while Jean Bricmont was at the Mathematics Department of Princeton University and Jean-Raymond Fontaine was at the Mathematics Department of Rutgers University. This work was supported by NSF Grant No. MCS 78-01885 at Princeton University and by NSF Grant No. PHY 78-15920 at Rutgers University
} 
Now we explain our definition of contours. Usually one defines contours as separating sites or groups of sites where different ground state configurations (or close to the ground states) occur. In our definition, contours separate blocks of spins, where the average of the spins over the block is close to the ground state value. That is, the average of $S_{i}^{1}$ in one block will be close to +1 and over the next block close to -1 .

Moreover, we let the size of the blocks depend on $\beta$ in order to get improved estimates, uniform in $\beta$ for $\alpha=e^{-c \beta}$. We estimate the probability of these contours with the infrared bounds. This requires reflection positivity but is different from the contour estimates of $[8,9]$. However, it is an exact analogue for lattice systems of the contour estimates in $P(\phi)_{2}$ models $[12,7]$.

We explain this further in Sect. 3. We show that in a lattice $\lambda \phi^{4}-\sigma \phi^{2}$ model there is a spontaneous magnetisation for suitable $\lambda, \sigma$ uniformly in the lattice spacing $a$. The limit $a \rightarrow 0$ is analogous to $\beta \rightarrow \infty$ in the anisotropic rotator. However the field theory case offers some simplification, and our proof is merely an adaptation to the lattice of [7]. On the other hand, the result is relevant for comparison with some numerical computations [1-3]. (See last remark in Sect. 3.)

\section{Anisotropic Rotators}

At each site $x \in \mathbb{Z}^{2}$ there is an $n$-component rotator $\mathbf{S}_{x} \in S^{n-1}$ of unit length. The Hamiltonian $H$ is

$$
-H=\sum_{\langle x y\rangle} \mathbf{S}_{x} \cdot \mathbf{S}_{y}+\alpha \sum_{\langle x y\rangle} S_{x}^{1} S_{y}^{1}
$$

where the sums run over nearest neighbour pairs in a finite box $\Lambda \subset \mathbb{Z}^{2}$ including suitable boundary conditions. We say that there is long range order for this system at inverse temperature $\beta$ is some translation invariant Gibbs state does not have the clustering property.

Theorem. There exist $K<\infty, c>0$ and $a \beta_{0}>0$ such that for $\alpha=(K / \beta) \exp (-c \beta)$ and all $\beta>\beta_{0}$ there is long range order.

In the proof, we consider for simplicity the case $n=2$ (two component rotator). The extension to any $n$ is explained in the remark at the end of this section.

Proof. We take $\Lambda$ to be a square of side $L$ :

$$
\Lambda=\left\{x \in \mathbb{Z}^{2} \mid 0 \leqq x_{1} \leqq L-1,0 \leqq x_{2} \leqq L-1\right\},
$$

and we put periodic boundary conditions in (2). Let $L=2^{p} b$, where $b$ is the side of a smaller box

$$
B=\left\{x \in \mathbb{Z}^{2} \mid 0 \leqq x_{1} \leqq b-1,0 \leqq x_{2} \leqq b-1\right\}
$$

which will be chosen (later) as a function of $\beta$. We let $p$ (i.e. $|\Lambda|)$ go to infinity via a sequence $p_{n}$ such that the sequence of periodic Gibbs states in $\Lambda_{n}$ converge. We shall prove that the limiting state does not cluster. We denote $B_{i}, i \in \mathbb{Z}^{2}$ the 
translate of $B_{0}$ by multiples of $b: B_{i}=B_{0}+\left(i_{1} b, i_{2} b\right)$, and define our contours via

the following characteristic functions (c.f.):
$\chi_{i}^{+}$: c.f. of the event $\sum_{x \in B_{i}} \cos \theta_{x} \geqq 0$ and $\sum_{x \in B_{i}} \frac{\cos ^{2} \theta_{x}}{|B|} \geqq 1 / 8$,

$\chi_{i}^{0}:$ c.f. of the event $\sum_{x \in B_{i}} \frac{\cos ^{2} \theta_{x}}{|B|}<1 / 8$,

$\chi_{i}^{-}$: c.f. of the event $\sum_{x \in B_{i}} \cos \theta_{x} \leqq 0$ and $\sum_{x \in B_{i}} \frac{\cos ^{2} \theta_{x}}{|B|} \geqq 1 / 8$.

Here $|B|=b^{2}$ is the number of sites in $B_{i}$. Obviously, $\chi_{i}^{+}+\chi_{i}^{0}+\chi_{i}^{-}=1 \forall i$.

We shall prove two estimates, for $\alpha=e^{-c \beta}$ and $b$ a suitable function of $\beta$ :

a)

$$
\begin{gathered}
\left\langle\chi_{i}^{0}\right\rangle_{\Lambda} \rightarrow 0 \quad \text { as } \beta \rightarrow \infty, \\
\text { b) }\left\langle\chi_{i}^{+} \chi_{j}^{-}\right\rangle_{\Lambda} \leqq 1 / 4-\delta, \quad \delta>0,
\end{gathered}
$$

uniformly in $\Lambda, i$, and $j$ as $\beta \rightarrow \infty$. Since

$$
\left\langle\chi_{i}^{+}\right\rangle_{A}=\left\langle\chi_{i}^{-}\right\rangle_{\Lambda}=(1 / 2)\left(1-\left\langle\chi_{i}^{0}\right\rangle_{\Lambda}\right),
$$

this implies long range order.

From now on, we suppress the index on the expectation values. The proof of b) starts in a standard way (see [7] for more details): we introduce

$$
1=\prod_{B_{i} \subset \Lambda}\left(\chi_{i}^{+}+\chi_{i}^{0}+\chi_{i}^{-}\right)
$$

and expand the product. For each term in the resulting sum, define a box $B_{i}$ to be irregular if either $\chi_{i}^{0}$ occurs for the box $B_{i}$ in that term or $\chi_{i}^{+} \chi_{j}^{-}$(or $\chi_{i}^{-} \chi_{j}^{+}$) occurs for some box $B_{j}$ that has one side in common with $B_{i}$. Define a contour to be a connected set of irregular boxes. So to each term we associate a set of contours, and every non-zero term must have a contour separating $i$ and $j$. So the proof is reduced to the following estimate on the probability of a contour $\gamma$ :

$$
\left\langle\prod_{i \in \gamma} \chi_{i}^{\varepsilon(i)}\right\rangle \leqq \exp (-\varrho|\gamma|)
$$

where $\varepsilon(i)=0, \pm 1$ depending on $\gamma$ and $\varrho$ is large enough to control the "entropy" (=the number of contours with given length and given origin) of the contours. Note that this "entropy" is a purely geometrical quantity that does not depend on $b$. So, letting $b$ depend on $\beta$ in order to get (3) with $\varrho$ large enough and uniform in $\beta$ will not affect the entropy estimate.

The proof of (3) which itself concludes the proof of b) above and the proof of a) above follow from the Schwartz inequality and the proposition below:

Proposition ("contour estimate"). Let $b=\exp \left(c^{\prime} \beta\right)$. Then

a) For $\alpha=(K / \beta) \exp (-c \beta)$ with $c<2 c^{\prime}, \beta$ large enough, and $\mathscr{A}$ any set of boxes $B_{i} \subset \Lambda$,

$$
\left\langle\prod_{B_{i} \in \mathscr{A}} \chi_{i}^{0}\right\rangle \leqq \exp \left(-\frac{(K-1)}{12}|\mathscr{A}|\right)
$$


b) There exists a $\lambda$ independent of $\beta$ and of $c^{\prime}$ such that for $\mathscr{B}$ a set of pairs of adjacent boxes and any $\alpha \geqq 0$,

$$
\left\langle\prod_{(i, j) \in \mathscr{B}} \chi_{i}^{+} \chi_{j}^{-}\right\rangle \leqq\left(c^{\prime} \lambda\right)^{|\mathscr{B}| / 2}
$$

Remarks. 1) Estimate b) does not depend on $\alpha$. Indeed it holds for $\alpha=0$. So we choose $c^{\prime}$ small enough in this estimate in order to have $\varrho$ large enough in (3). This fixes the $\beta$ dependence of $b$. Then in a) we have to take $\alpha=(K / \beta) \exp (-c \beta)$ with $c<2 c^{\prime}$, that is $\alpha$ is constrained by the choice of $c^{\prime}$ not to be too small. The closer $c$ is from $2 c^{\prime}$ the larger $\beta$ has to be in order to get (4).

2) The basic ideas behind the estimates are as follows:

a) If $\sum_{x \in B_{i}} \frac{\cos ^{2} \theta_{x}}{|B|}$ is small $(<1 / 8)$, then $\sum_{\langle x y\rangle \subset B} \frac{\cos \theta_{x} \cos \theta_{y}}{|B|}$ is small, but this is unlikely because the Hamiltonian contains a term "favoring" $\cos \theta_{x} \cos \theta_{y}$. However, for this to be effective, we need $|B|$ very large if $\alpha$ is small : $\alpha \beta>1 /|\mathrm{B}|$ or $c<2 c^{\prime}$.

b) On the other hand, $\chi_{i}^{+} \chi_{j}^{-}$is unlikely because there is a "forbidden" region $\sum_{x \in B_{i}} \frac{\cos ^{2} \theta_{x}}{|B|} \leqq 1 / 8$. So, on the average, there is a jump between $B_{i}$ and $B_{j}$,

$$
\left(1 /|B|^{2}\right) \sum_{x \in B_{i}, y \in B_{j}}\left(\cos \theta_{x}-\cos \theta_{y}\right)^{2}
$$

cannot be too small; but, if $b$ is not too large, this is unlikely because of the infrared bounds that roughly say (in two dimensions)

$$
\left\langle\left(\cos \theta_{x}-\cos \theta_{y}\right)^{2}\right\rangle \leqq(1 / \beta) \log |x-y| \text {. }
$$

For $b=e^{c / \beta}$ and $x, y$ in adjacent boxes, this is of order $c^{\prime}$ (chosen to be small).

We start by proving part a) of the proposition. The proof of part b) will follow after three lemmas.

Proof of Part a). We use the method of [4], but chessboard estimates could be used instead.

$$
\begin{aligned}
\left\langle\prod_{i \in \mathscr{A}} \chi_{i}^{0}\right\rangle= & \left\langle\prod _ { i \in \mathscr { A } } \left(\chi_{i}^{0} \exp \left(\frac{K-1}{|B|} \sum_{\langle x y\rangle \subset B_{i}} \cos \theta_{x} \cos \theta_{y}\right)\right.\right. \\
& \left.\left.\cdot \exp \left(-\left(\frac{K-1}{|B|}\right) \sum_{\langle x y\rangle \subset B_{\imath}} \cos \theta_{x} \cos \theta_{y}\right)\right)\right\rangle .
\end{aligned}
$$

Since $\cos \theta_{x} \cos \theta_{y} \leqq(1 / 2)\left(\cos ^{2} \theta_{x}+\cos ^{2} \theta_{y}\right)$ and \# nearest neighbour pairs in $B=2|B|$, we have

$$
\text { right hand side of } \begin{aligned}
(6) \leqq & \exp \left(\frac{(K-1)}{4}|\mathscr{A}|\right) \\
& \cdot\left\langle\exp -\left(\frac{K-1}{|B|}\right) \sum_{\langle x y\rangle \subset B_{i}} \cos \theta_{x} \cos \theta_{y}\right\rangle .
\end{aligned}
$$

Then, by the method of [4], this is less than

$$
\exp \left(\frac{(K-1)}{4}|\mathscr{A}|\right) \exp \left(-(K-1)|\mathscr{A}|\left\langle\cos \theta_{x} \cos \theta_{y}\right\rangle^{\prime}\right)
$$


as long as $\alpha \beta \geqq K|B|^{-1} ;\langle\rangle^{\prime}$ is the expectation value with respect to the measure

$$
\begin{gathered}
Z^{-1} \exp \left[\beta\left(\cos \left(\theta_{x}-\theta_{y}\right)+|B|^{-1} \cos \theta_{x} \cos \theta_{y}\right)\right] d \theta_{x} d \theta_{y} ; \\
\lim _{\beta \rightarrow \infty}\left\langle\cos \theta_{x} \cos \theta_{y}\right\rangle^{\prime} \geqq 1 / 2 .
\end{gathered}
$$

So we get, for $\beta$ large enough,

$$
\begin{aligned}
\left\langle\prod_{i \in \mathscr{A}} \chi_{i}^{0}\right\rangle & \leqq \exp ((K-1)|\mathscr{A}| / 4-(K-1)|\mathscr{A}| / 3) \\
& =\exp ((K-1)|\mathscr{A}| / 12) . \square
\end{aligned}
$$

Now we state and prove three lemmas that are needed for part $b$ ).

\section{Lemma 1.}

$$
\chi_{i}^{+} \chi_{j}^{-} \leqq\left(4 /|B|^{2}\right) \sum_{\substack{x \in B_{i} \\ y \in B_{j}}}\left(\cos \theta_{x}-\cos \theta_{y}\right)^{2} .
$$

Proof. If $\chi^{+} \chi^{-}=1$, then

$$
|B|^{-1}\left(\sum_{x \in B_{i}} \cos ^{2} \theta_{x}+\sum_{y \in B_{j}} \cos ^{2} \theta_{y}\right) \geqq 1 / 4,
$$

and

$$
-\sum_{x \in B_{i}, y \in B_{J}} \cos \theta_{x} \cos \theta_{y} \geqq 0 .
$$

From Lemma 1, follows the formula:

$$
\left\langle\prod_{(i, j) \in \mathscr{B}} \chi_{i}^{+} \chi_{j}^{-}\right\rangle \leqq\left(4 /|B|^{2}\right)^{|\mathscr{B}|} \sum_{\mathscr{F}}\left\langle\prod_{\left(x_{i}, y_{j}\right) \in \mathscr{F}}\left(\cos \theta_{y_{i}}-\cos \theta_{y_{j}}\right)^{2}\right\rangle,
$$

where the sum runs over all families $\mathscr{F}$ of pairs $\left(x_{i}, y_{j}\right)$ where we choose some $x_{i} \in B_{i}$ and some $y_{j} \in B_{j}$ for each pair $(i, j) \in \mathscr{B}$; notice that $|\mathscr{F}|=|\mathscr{B}|$ and that the sum runs over $|B|^{2|\mathscr{B}|}$ families.

Now we consider the subset $\mathscr{B}^{\prime}$ of $\mathscr{B}$ of pairs $(i, j)$ such that $B_{i}$ and $B_{j}$ share a vertical side. Without loss of generality, we assume that a majority of pairs in $\mathscr{B}$ have this property:

$$
\left|\mathscr{B}^{\prime}\right| \geqq|\mathscr{B}| / 2,
$$

otherwise, change vertical into horizontal. Since $\chi^{+} \chi^{-} \leqq 1$, we have

$$
\left\langle\prod_{(i, j) \in \mathscr{B}} \chi_{i}^{+} \chi_{j}^{-}\right\rangle \leqq\left\langle\prod_{(i, j) \in \mathscr{B}^{\prime}} \chi_{i}^{+} \chi_{j}^{-}\right\rangle
$$

and we apply (7) to the right hand side of (9).

Next we use chessboard estimates on the right hand side of (7). For this we define the strips

$$
\begin{aligned}
& S_{1}=\left\{x \in \mathbb{Z}^{2} \mid 0 \leqq x_{1} \leqq 2 b-1,0 \leqq x_{2} \leqq L\right\} \\
& S_{2}=\left\{x \in \mathbb{Z}^{2} \mid 0 \leqq x_{1} \leqq L, 0 \leqq x_{2} \leqq b-1\right\}
\end{aligned}
$$

and the sublattice

$$
\mathscr{L}=\left\{\left(n_{1} 2 b, n_{2} b\right) \mid n_{1}, n_{2} \in \mathbb{N}, 0 \leqq n_{1} \leqq 2^{(p-1)}-1,0 \leqq n_{2} \leqq 2^{p}-1\right\} .
$$


The subsets $\mathscr{L}_{v} \subset \mathscr{L}, v=0,1,2,3$ are defined by $\mathscr{L}_{0}: n_{1}, n_{2}$ even; $\mathscr{L}_{1}: n_{1}$ even $n_{2}$ odd; $\mathscr{L}_{2}: n_{1}$ odd $n_{2}$ even; $\mathscr{L}_{3}: n_{1}, n_{2}$ odd. For $x \in B_{(0,0)} \cup B_{(1,0)}$, we define

$$
\begin{aligned}
& \varrho_{0}(x)=x, \\
& \varrho_{1}(x)=\left(2 b-x_{1}, x_{2}\right), \\
& \varrho_{2}(x)=\left(x_{1}, b-x_{2}\right), \\
& \varrho_{3}(x)=\left(\varrho_{1} \circ \varrho_{2}\right)(x)=\left(2 b-x_{1}, b-x_{2}\right) .
\end{aligned}
$$

Finally, for any $x \in \Lambda$ there is a unique $i \in \mathscr{L}$ such that $x-i \in B_{(0,0)} \cup B_{(1,0)}$. We define $\varrho_{v}(x) \equiv \varrho_{v}(x-i), v=0,1,2,3$.

Lemma 2 (Chessboard Estimates). Let $\mathscr{F}$ be a term in the right hand side of formula (7) applied to $\mathscr{B}^{\prime}$. Then

$$
\begin{aligned}
& \left\langle\prod_{(x, y) \in \mathscr{F}}\left(\cos \theta_{x}-\cos \theta_{y}\right)^{2}\right\rangle \\
& \quad \leqq\left(c^{\prime}\right)^{|\mathscr{F}|} \prod_{(x, y) \in \mathscr{F}} \cdot\left\langle\exp \left[\left(c^{\prime}\right)^{-1 / 2} \sum_{v=0}^{3} \sum_{s \in \mathscr{L}_{v}}\left(\cos \theta_{\varrho_{v}(x)+s}-\cos \theta_{\varrho_{v}(y)+s}\right)\right]\right\rangle^{\frac{2(B)}{|\Lambda|}} .
\end{aligned}
$$

Proof. First we use $u^{2} \leqq\left(e^{u}+e^{-u}\right) / 2$, with $u=\sqrt{c^{\prime}}\left(\cos \theta_{x}-\cos \theta_{y}\right)$. So

$$
\begin{aligned}
& \prod_{(x, y) \in \mathscr{F}}\left(\cos \theta_{x}-\cos \theta_{y}\right)^{2} \\
& \leqq\left(c^{\prime}\right)^{|\mathscr{F}|} 2^{-|\mathscr{F}|} \sum_{\sigma_{x y}= \pm 1}\left\langle\prod_{(x, y) \in \mathscr{F}} \exp \left[\left(c^{\prime}\right)^{-1 / 2} \sigma_{x y}\left(\cos \theta_{x}-\cos \theta_{y}\right)\right]\right\rangle .
\end{aligned}
$$

We refer to [8] for our use of chessboard estimates : first let $\mathscr{A}_{0}$ be the functions depending on the spins in $S_{1}$ and $\varrho=\varrho_{1}$. Then use formula (4.3) of [8]. Repeat the operation with $S_{2}$ and $\varrho=\varrho_{2}$. Note that $\varrho_{v}^{2}=1 \forall v$.

Finally, we use the invariance of $\left\langle>\right.$ under $\cos \theta_{x} \rightarrow-\cos \theta_{x}$ to estimate the sum over $\sigma_{x y}$.

In order to state the last lemma (Infrared bounds) we define $\langle\cdot\rangle_{A}^{0}$ as the expectation value in the massless Gaussian field in $\Lambda$ with periodic boundary conditions. This is well defined only for expressions involving differences of fields $\left(\phi_{x}-\phi_{y}\right)$ because of the boundary conditions. Explicitly:

where

$$
\left\langle\phi_{0}\left(\phi_{x}-\phi_{y}\right)\right\rangle_{\Lambda}^{0}=|\Lambda|^{-1} \sum_{\substack{k \in \Lambda^{*} \\ k \neq 0}} \frac{\exp (i k x)-\exp (i k y)}{2\left(1-\cos k_{1}+1-\cos k_{2}\right)},
$$

$$
\Lambda^{*}=\left\{k=\left(k_{1}, k_{2}\right)=\left(2 \pi \kappa_{1} / L, 2 \pi \kappa_{2} / L\right) \mid \kappa_{i} \in \mathbb{N}, 0 \leqq \kappa_{i} \leqq L-1\right\} .
$$

\section{Lemma 3 (Infrared or Grad- $\phi$ Bounds).}

$$
\begin{aligned}
& \left\langle\exp \left[\left(c^{\prime}\right)^{-1 / 2} \sum_{i=1}^{n}\left(\cos \theta_{x_{i}}-\cos \theta_{y_{i}}\right)\right]\right\rangle \\
& \quad \leqq \exp \left[\left(2 \beta \mathrm{c}^{\prime}(1+\alpha)\right)^{-1} \sum_{i, j=1}^{n}\left\langle\left(\phi_{x_{i}}-\phi_{y_{i}}\right)\left(\phi_{x_{j}}-\phi_{y_{j}}\right)\right\rangle_{\Lambda}^{0}\right] .
\end{aligned}
$$


Proof. This is just a restatement of Theorem 2.1 of [10] or formula (4.11) of [8], after summation by parts.

Proof of Proposition, Part b). We apply successively Eq. (9) to $\left\langle\prod_{(i, j) \in \mathscr{B}} \chi_{i}^{+} \chi_{j}^{-}\right\rangle$, then Lemma 1 in the form of Eq. (7) to the right hand side of (9); Lemma 2 is applied to each $\mathscr{F}$ in (7) and finally Lemma 3 to the right hand side of (10), where the sum over $i=1 \ldots n$ is replaced by the sum over $v=0 \ldots 3, s \in \mathscr{L}_{v}$ and $x_{i}=\varrho_{v}(x)+s$, $y_{i}=\varrho_{v}(y)+s$. We get (using $|\mathscr{F}|=\left|\mathscr{B}^{\prime}\right|$ )

$$
\begin{aligned}
\left\langle\prod_{(i, j) \in \mathscr{B}} \chi_{i}^{+} \chi_{j}^{-}\right\rangle \leqq & \left(4 c^{\prime} /|B|^{2}\right)^{\left|\mathscr{B}^{\prime}\right|} \\
& \cdot \prod_{\mathscr{F}} \prod_{(x, y) \in \mathscr{F}} \exp \left[\left(2|B| /|\Lambda| 2 \beta c^{\prime}(1+\alpha)\right)\right. \\
& \left.\cdot \sum_{v, v^{\prime}=0}^{3} \sum_{\substack{s \in \mathscr{L}_{v} \\
s^{\prime} \in \mathscr{L}_{v^{\prime}}}}\left\langle\left(\phi_{\varrho_{v}(x)+s}-\phi_{\varrho_{v}(y)+s}\right)\left(\phi_{\varrho_{v^{\prime}}(x)+s}-\phi_{\varrho_{v^{\prime}}(y)+s}\right)\right\rangle_{\Lambda}^{0}\right],
\end{aligned}
$$

Now we perform explicit cancellations in the sums over $v, v^{\prime}, s, s^{\prime}$. Let us consider $v=v^{\prime}=0$. The other cases are treated similarly. By periodicity the sum over $s^{\prime}$ gives $|\Lambda| / 8|B|$ times the sum over $s$ for $s^{\prime}=0$. If we write this explicitly,

$$
\left\langle\left(\phi_{x+s}-\phi_{y+s}\right)\left(\phi_{x}-\phi_{y}\right)\right\rangle_{\Lambda}^{0}=\left|\Lambda^{*}\right|^{-1} \sum_{\substack{k \in \Lambda^{*} \\ k \neq 0}} \frac{\exp (i k s)(2-2 \cos k(x-y))}{2\left(1-\cos k_{1}+1-\cos k_{2}\right)} .
$$

The sum over $s \in \mathscr{L}_{0}$ will be zero unless

$$
k \in(\bar{B})^{*}=\left\{k=\left(\frac{2 \pi n_{1}}{4 b}, \frac{2 \pi n_{2}}{2 b}\right) \mid n_{i} \in \mathbb{N}, 0 \leqq n_{1} \leqq 4 b-1,0 \leqq n_{2} \leqq 2 b-1\right\}
$$

the dual of

$$
\bar{B}=\left\{x \in \mathbb{Z}^{2} \mid 0 \leqq x_{1} \leqq 4 b-1,0 \leqq x_{2} \leqq 2 b-1\right\}
$$

If $k \in(\bar{B})^{*}$, the sum over $s$ of $e^{i k s}$ is just $|\Lambda| / 8|B|$; therefore the sum over $s \in \mathscr{L}_{0}$ of

$$
\left\langle\left(\phi_{x}-\phi_{y}\right)\left(\phi_{x+s}-\phi_{y+s}\right)\right\rangle_{A}^{0}=\left\langle\left(\phi_{x}-\phi_{y}\right)^{2}\right\rangle_{\bar{B}}^{0}
$$

diverges $\log$ arithmically with $\bar{B}$, i.e. it is $O(\log b)=O\left(c^{\prime} \beta\right)$. Therefore, the argument of the exponential in (11) is $O(1)$. The sum over $\mathscr{F}$ involves exactly $B^{2\left|\mathscr{B}^{\prime}\right|}$ terms; to finish the proof we can take $\lambda$ in (5) to equal $4 \exp O(1)$, and we use $\left|\mathscr{B}^{\prime}\right| \geqq|\mathscr{B}| / 2$.

Remark. For $n$ component rotators, the only non-trivial extension concerns the estimate on $\chi^{0}$ [part a)] where we use (implicitly) correlation inequalities known only for $n=2$. However, we can either use reflection positivity and a thermodynamic estimate or extend the proof of Theorem 3a in [4] to the case $s_{x}^{1} s_{y}^{1} \leqq 1 / 4 e n^{2}$ and then extend it to blocks in the same way that we extended here the proof of Theorem 2 of [4]. However, we have to replace $1 / 8$ in the definition of $\chi^{0}$ (and of $\chi^{+}, \chi^{-}$) by $1 / 8 e n^{2}$. 


\section{Lattice Field Theory}

We can consider the model given, in a finite box $\Lambda \subset \mathbb{Z}^{2}$ by

$$
H=\frac{1}{2} \sum_{\langle x y\rangle \subset A}^{p}\left(\phi_{x}-\phi_{y}\right)^{2}+(\lambda / 4) \sum_{x \in A} \phi_{x}^{4}-(\sigma / 2) \sum_{x \in \Lambda} \phi_{x}^{2}, \quad \phi_{x} \in \mathbb{R}
$$

we impose periodic b.c. on $\Lambda$. The Gibbs measure $d \mu_{\Lambda}$

$$
d \mu_{\Lambda}=Z_{\Lambda}^{-1} \exp \left(-H_{\Lambda}\right) \prod_{x \in \Lambda} d \phi_{x} .
$$

Since $\beta$ can be absorbed into $\lambda, \sigma$ by a change of scale it is set equal to one.

Again we consider states that are limits of $d \mu_{\Lambda}$ as $\Lambda \rightarrow \mathbb{Z}^{2}$ through a suitable sequence and we speak of long range order as before.

Theorem. There exists $a c>0$ and a $\bar{\lambda}>0$ such that for

$$
\sigma=(3 / 4 \pi) \lambda|\log \lambda|+c \lambda,
$$

then for all $\lambda \leqq \bar{\lambda}$ there is long range order.

Remarks. 1) The proof is patterned after that of [7] which simplifies the proof in [12] of phase transitions for continuum $\left(\phi^{4}\right)_{2}$. The relationship between a weak coupling $(\lambda$ small $)$ lattice field theory and the continuum limit is made explicit if we reparametrize $\lambda, \sigma$ as follows: let

$$
\lambda=\lambda_{0} \delta^{2}, \quad \sigma=(3 / 4 \pi) \lambda_{0} \delta^{2}\left|\log \delta^{2}\right|+\sigma_{0} \delta^{2},
$$

where

$$
\sigma_{0}=(3 / 4 \pi) \lambda_{0}\left|\log \lambda_{0}\right|+c \lambda_{0} .
$$

Think of $\delta$ as a lattice spacing. Then we can rewrite

$$
\begin{aligned}
(\lambda / 4) \phi_{x}^{4}-(\sigma / 2) \phi_{x}^{2}= & \left(\lambda_{0} / 4\right) \delta^{2}: \phi_{x}^{4}:_{\delta, 1} \\
& -\left(\left(\sigma_{0}-1-O\left(\lambda_{0}\right)\right) / 2\right) \delta^{2}: \phi_{x}^{2}:_{\delta, 1} \\
& +(1 / 2) \delta^{2} \phi_{x}^{2}+\text { const },
\end{aligned}
$$

where the Wick-ordering $::_{\delta, 1}$, is with respect to a lattice free field of mass 1 and lattice spacing $\delta$ :

$$
\begin{aligned}
: \phi_{x}^{4}:_{\delta, 1} & =\phi_{x}^{4}-6 c_{00}^{1, \delta} \phi_{x}^{2}+\text { const }, \\
c_{00}^{m, \delta} & =(2 \pi)^{-2} \int_{-\pi / \delta}^{\pi / \delta} \frac{d^{2} K}{2\left(1-\cos K_{1} \delta+1-\cos K_{2} \delta\right) / \delta^{2}+m^{2}} .
\end{aligned}
$$

As $\delta \rightarrow 0$, one computes that

$$
c_{00}^{1, \delta} \simeq(1 / 4 \pi)\left|\log \delta^{2}\right|+O(1) .
$$

The $1 / 4 \pi$ and the factor 6 in the Wick ordering explain the $3 / 4 \pi$ in formula (12) for $\sigma$. The $O(1)$ gives the $O\left(\lambda_{0}\right)$ in (14). In (15) we recognize the formula for a lattice approximation of a field theory (FT)

$$
\left(\lambda_{\mathrm{FT}} / 4\right): \phi^{4}:_{m^{2}}-\left(\sigma_{\mathrm{FT}} / 2\right): \phi^{2}:_{m^{2}}+\frac{m^{2}}{2}: \phi^{2}:_{m^{2}},
$$


where

$$
m^{2}=1, \quad \lambda_{\mathrm{FT}}=\lambda_{0}, \quad \sigma_{\mathrm{FT}}=\sigma_{0}-1-\left(\lambda_{0}\right) .
$$

If we choose $c$ in (13) large and $\lambda_{0}$ small, we can make $\sigma_{\mathrm{FT}} / \lambda_{\mathrm{FT}}$ as large as we need in order to be, for the continuum field theory, in the two phase region $[7,12]$. So the theorem can be viewed exactly as a version of this result on a lattice, which holds uniformly in the lattice spacing $\delta$.

2) In [6] it is proved that, if $c$ in (13) is sufficiently negative, then the correlation functions decay exponentially for $\lambda$ sufficiently small. This combined with Theorem 2 implies that for this model the critical $\sigma$ satisfies:

$$
\sigma_{c}(\lambda)=(3 / 4 \pi) \lambda|\log \lambda|
$$

to leading order in $\lambda$ as $\lambda \rightarrow 0$, with an error of order $\lambda$. Thus $\sigma_{c}(\lambda)$ can be defined as the sup over $\lambda$ 's such that the correlation functions decay exponentially. This is the two dimensional analogue of the estimates of [5] on $\sigma_{c}(\lambda)$.

3) The main estimates that are used in the proof and what follows from field theoretic results are stability bounds on the partition function $[12,7]$.

Proof. We indicate the differences with the proof of Theorem 1. Also all the difficult estimates are taken from [7]. The main difference with the anisotropic rotator lies in the definition of $\chi^{+}, \chi^{-}, \chi^{0}: \chi_{i}^{+}:$c.f. of the event $|B|^{-1} \sum_{x \in B_{i}} \phi_{x} \geqq K$,

$$
\begin{aligned}
& \chi_{i}^{-}: \text {c.f. of the event }(B)^{-1} \sum_{x \in B} \phi_{x} \leqq-K, \\
& \chi_{i}^{0} \text { : c.f. of the event }(B)^{-1}\left|\sum_{x \in B} \phi_{x}\right| \leqq K .
\end{aligned}
$$

Here $|B|=b^{2}$ will be chosen of order $\lambda^{-1}$ (i.e. $\delta^{-2}$ if we refer to Remark 1 above) and $K$ is a large number to be chosen later. Now, following the proof of Theorem 1, we have to estimate

$$
\left\langle\prod_{(i, j) \in \mathscr{B}} \chi_{i}^{(+)} \chi_{j}^{-}\right\rangle \leqq \exp (-2 K|\mathscr{B}|)\left\langle\exp \left[\sum_{(i, j) \in \mathscr{B}}(B)^{-1}\left(\sum_{x \in B_{i}} \phi_{x}-\sum_{y \in B_{J}} \phi_{y}\right)\right]\right\rangle .
$$

We write

$$
\sum_{x \in B_{\imath}} \phi_{x}-\sum_{y \in B_{j}} \phi_{y}=\sum_{x \in B_{\imath}} \phi_{x}-\phi_{x^{\prime}}
$$

by making a one to one correspondence between points of $B_{i}$ and $B_{j}$.

The expectation value is then shown to be $O(1)^{|\mathscr{B}|}$ by the infrared (or grad- $\phi$ ) bounds using Lemma 3. (It is only easier because we do not have to perform cancellations as in the proof of Theorem 1, since we do not use chessboard estimates here.)

We have also to bound

$$
\left\langle\prod_{i \in \mathscr{A}} \chi_{i}^{0}\right\rangle_{\Lambda} \leqq \exp \left(1 / 2 \sigma_{\mathrm{FT}} K|\mathscr{A}|\right)\left\langle\exp \left[-\left(\sigma_{\mathrm{FT}} / 2\right) \sum_{i \in \mathscr{A}}\left(\sum_{x \in B_{i}} \frac{\phi_{x}}{|B|}\right)^{2}\right]\right\rangle_{\Lambda},
$$

with $\sigma_{\mathrm{FT}}$ given by (17). 
Using chessboard estimates [7], the expectation value in the right hand side of (19) is bounded by

$$
\left(\left\langle\exp \left[-\left(\sigma_{\mathrm{FT}} / 2\right) \sum_{i \in \mathscr{L}}\left(\sum_{x \in B_{i}} \frac{\phi_{x}}{|\mathrm{~B}|}\right)^{2}\right]\right\rangle_{\Lambda}^{|B| /|\Lambda|}\right)^{|\mathscr{A}|} .
$$

Now, using the method of [7, proof of Lemma 7.4], one can show that $Z_{\Lambda}^{|B| /|\Lambda|}$ diverges uniformly in $|\Lambda|$ as $\lambda_{0}$ in (14) goes to zero. If we think of the lattice approximation $|\Lambda| /|B|$ is the size of the box in the continuum limit and $\lambda_{0}=\lambda_{\mathrm{FT}}$. So as $\lambda_{0} \rightarrow 0$, the factor $\exp \left(-\left(\lambda_{0} / 4\right) \phi^{4}\right)$ that guarantees the stability of $Z_{\Lambda}$ disappears and the factor $\exp \left((\sigma / 2) \phi^{2}\right)$ remains. This factor produces, for suitable $c$, a negative effective square mass.

On the other hand this factor is compensated in the numerator by the $\exp \left(-\left(\sigma_{\mathrm{FT}} / 2\right) \ldots\right)$ in $(20)$ as shown in [7, Eq. (7.31)]. One can write

$$
\sum_{i \in \mathscr{L}}\left(\sum_{x \in B_{i}} \frac{\phi_{x}}{|B|}\right)^{2}=\sum_{i \in \mathscr{L}}:\left(\sum_{x \in B_{i}} \frac{\phi_{x}}{\left|B_{i}\right|}\right)^{2}:_{\delta, 1}+\sum_{i \in \mathscr{L}}\left\langle\left(\sum_{x \in B_{i}} \phi_{x} /|B|\right)^{2}\right\rangle_{\delta, 1},
$$

where \langle\rangle$_{\delta, 1}$ is the lattice Gaussian with covariance defined in (15). The second term contains $|\Lambda| /|B|$ terms each of which is bounded (as $\delta \rightarrow 0$ it approaches $\left\langle\left(\int_{\square} \phi(x) d^{2} x\right)^{2}\right\rangle, \square=$ unit box $)$; the first term compensates the $\left(\sigma_{\mathrm{FT}} / 2\right) \sum_{x}: \phi_{x}^{2}:_{1}$ in (14) and (16). The net result is that the numerator is uniformly bounded in $\delta$ (or in our fixed lattice spacing notation in $\lambda$ for $\lambda \leqq \bar{\lambda}$ ). So $(20)$ is bounded by $(\varepsilon)^{|\mathscr{A}|}$ with $\varepsilon$ as small as we need for $\lambda_{0}$ small enough. Now we first choose $K$ large enough so that the bound (18) makes $\chi_{i}^{+} \chi_{j}^{-}$sufficiently unlikely. Then we choose $\varepsilon$ sufficiently small so that $\varepsilon \exp \left(\frac{1}{2} \sigma_{\mathrm{FT}} K\right)$ is small $\left(=\varepsilon^{\prime}\right)$ and therefore $\prod_{i \in \mathscr{A}} \chi_{i}^{0} \leqq\left(\varepsilon^{\prime}\right)^{|\mathscr{A}|}$.

Remarks. 1. The main technical difference between the proof in the $\lambda \phi^{4}$ and anisotropic rotator cases is that in the $\lambda \phi^{4}$ case we have strong stability bounds. This allows us to get the correct constant $(=3 / 4 \pi)$ in $\sigma_{c}(\lambda) \simeq c \lambda \log \lambda$, while the corresponding $c$ in the anisotropic rotator $(\alpha=\exp (-c \beta))$ is certainly not the best possible. In that case we do not have analogous stability bounds.

2. As we mentioned earlier, our results combined with the lower bound on $\sigma_{c}(\lambda)$ in [6] shows that

$$
\sigma_{c}(\lambda) \simeq(3 / 4 \pi) \lambda|\log \lambda|+O(\lambda)
$$

as $\lambda \rightarrow 0$. It was pointed out in [3] that a real space renormalization group calculation predicts a curve of this type for $\sigma_{c}(\lambda)$ when $\lambda \rightarrow 0$. This has to be contrasted with results predicted by a Kadanoff-Migdal transformation [2] which, in the same situation, led to a linear dependence of $\sigma_{c}$ on $\lambda$ (see also [1]). So our result (which could be guessed any way by field theoretic methods) settles the question in favor of the real space renormalization group calculation. However, in that approach the constant multiplying $\lambda \ln \lambda$ seems to be too large by a factor 5 [3].

Acknowledgements. We thank Jürg Fröhlich and Charles-Edouard Pfister for useful discussions and Paul Beale for his correspondence and for letting us know his results prior to publication. 


\section{References}

1. Baker, G.A., Bishop, A.R.: Order-disorder displacive crossover in a structural phase transition model. Los Alamos (preprint)

2. Beale, P., Sarker, S., Krumhansl, J.A.: A renormalisation group study of crossover in structural phase transitions. Phys. Rev. B24, 266 (1981)

3. Beale, P.: Critical and crossover behavior of the two dimensional $\phi^{4}$ model on a lattice. Phys. Rev. B24, 6711 (1981) and private communication

4. Bricmont, J., Fontaine, J.-R. : Correlation inequalities and contour estimates. J. Stat. Phys. 26, 745 (1981)

5. Bricmont, J., Fontaine, J.-R.: Perturbation about the mean field critical point. Commun. Math. Phys. 86, 337-362 (1982)

6. Brydges, D., Fröhlich, J., Spencer, T.: The random walk representation of classical spin systems and correlation inequalities. Commun. Math. Phys. 83, 123 (1982)

7. Fröhlich, J. : Phase transitions, Goldstone bosons and topological superselection rules. Acta Phys. Austriaca Suppl. XV, 133 (1976)

8. Fröhlich, J., Israel, R., Lieb, E., Simon, B.: Phase transitions and reflection positivity. ,I. General theory and long range models. Commun. Math. Phys. 62, 1 (1978)

9. Fröhlich, J., Lieb, E.: Phase transitions in anisotropic lattice spin systems. Commun. Math. Phys. 60, $233(1978)$

10. Fröhlich, J., Simon, B., Spencer, T.: Infrared bounds, phase transitions, and continuous symmetry breaking. Commun. Math. Phys. 50, 79 (1976)

11. Fröhlich, J., Spencer, T.: The Kosterlitz-Thouless transition in the two-dimensional abelian spin systems and the Coulomb gas. Commun. Math. Phys. 81, 527 (1981)

12. Glimm, J., Jaffe, A., Spencer, T.: Phase transitions for $\phi_{2}^{4}$ quantum fields. Commun. Math. Phys. 45, 203 (1975)

13. Kunz, H., Pfister, C.-E., Vuillermot, P.A.: Inequalities for some classical spin vector models. J. Phys. A 91673 (1976); Phys. Lett. 54A, 428 (1975)

14. Malyshev, S.: Phase transitions in classical Heisenberg ferromagnets with arbitrary parameter of anisotropy. Commun. Math. Phys. 40, 75 (1975)

Communicated by T. Spencer

Received August 1, 1982 
